\title{
CBG: a cortisol reservoir rather than a transporter
}

\author{
Marie-Pierre Moisan
}

The Review by Perogamvros et al. (Regulation of cortisol bioavailability-effects on hormone measurement and action. Nat. Rev. Endocrinol. 8, 717-727; 2012) provides an overview of the regulation of corticosteroid bioavailability and its consequences on the assessment of the hypothalamicpituitary-adrenal (HPA) axis, including the description of various approaches for evaluating the function of the HPA axis in patients. ${ }^{1}$ I first challenge their assertion that corticosteroid binding protein (CBG) is a transporter protein. Second, I describe transcriptomic analysis as another novel and promising method to assess HPA axis function.

In the Review, CBG is described as "the most important transporter for natural glucocorticoids". The argument put forward is that cortisol is hydrophobic and thus needs CBG to circulate in blood. However, firstly, in CBG-deficient patients cortisol circulates normally. ${ }^{2}$ Secondly, CBG is not present in many species, such as teleost fish, in which cortisol circulates. ${ }^{3-4}$ Thirdly, circulating aldosterone is equally hydrophobic and does not have a specific binding protein. Fourthly, a contradiction exists with the argument that the observed decrease in CBG levels, for example during sepsis, provides an increased supply of free cortisol to organs. In my opinion, the transport of glucocorticoids is ensured by albumin. Indeed, glucocorticoids are bound mainly to albumin in CBG-deficient patients or mice, ${ }^{5-6}$ and albumin is present in the blood of all vertebrates.

Why then did CBG appear during evolution and what is its specific role? As pointed out by Perogamvros et al., CBG is a delivery molecule that enables local release of massive amounts of cortisol at sites of inflammation by virtue of its SERPIN structure. Additionally, our studies in CBGdeficient mice demonstrate that the presence of CBG is necessary to mount a normal stress response. ${ }^{6-7}$ Contrary to what one might have expected, CBG deficiency leads to signs of hypocortisolism rather than hypercortisolism: free cortisol and corticosterone levels are normal under resting conditions and suboptimal after stress. Thus, the main, and perhaps sole, function of CBG seems to be the retention in blood of a circulating glucocorticoid reserve readily available in case of an emergency.

Perogamvros et al. report the necessity of measuring free rather than total cortisol levels to assess HPA axis function in patients. Although this approach would be progress, knowledge of free cortisol levels does not answer the question of whether cortisol exerts its actions normally or not. Indeed, free cortisol levels could be elevated, but if its receptors are desensitized cortisol action will not be effective. Cortisol acts on cells by binding to specific receptors that are transcription factors modulating the expression of target genes. These target genes have either glucocorticoid response elements in their promoter sequence or other DNA motifs for interfering transcription factors, such as NFkB. These properties are used in transcriptomic analyses, in which the level of global gene expression is examined followed by the use of promotersequence-based bioinformatic tools to identify glucocorticoid targets. ${ }^{8}$ This approach for assessing HPA axis function has been used successfully in stress research, ${ }^{9-10}$ requires a single $5 \mathrm{ml}$ blood collection and its cost is reducing.
Institut National de la Recherche Agronomique, Nutrition and Integrative Neurobiology, Université Bordeaux ,UMR1286, 146 rue Léo Saignat, 33076 Bordeaux cedex, France. mpmoisan@bordeaux.inra.fr

doi:10.1038/nrendo.2012.134-c1

Competing interests

The author declares no competing interests.

1. Perogamvros, I., Ray, D. W. \& Trainer, P. J. Regulation of cortisol bioavailability-effects on hormone measurement and action. Nat. Rev. Endocrinol. 8, 717-727 (2012).

2. Gagliardi, L., Ho, J. T. \& Torpy, D. J. Corticosteroidbinding globulin: the clinical significance of altered levels and heritable mutations. Mol. Cell. Endocrinol. 316, 24-34 (2010).

3. Breuner, C. W. \& Orchinik, M. Plasma binding proteins as mediators of corticosteroid action in vertebrates. J. Endocrinol. 175, 99-112 (2002).

4. Moisan, M. P. Genotype-phenotype associations in understanding the role of corticosteroid-binding globulin in health and disease animal models. Mol. Cell. Endocrinol. 316, 35-41 (2010).

5. Emptoz-Bonneton, A., et al. Novel human corticosteroid-binding globulin variant with low cortisol-binding affinity. J. Clin. Endocrinol. Metab. 85, 361-367 (2000).

6. Richard, E. M. et al. Plasma transcortin influences endocrine and behavioral stress responses in mice. Endocrinology 151, 649-659 (2010).

7. Minni, A. M. et al. Critical role of plasma corticosteroid-binding-globulin during stress to promote glucocorticoid delivery to the brain: impact on memory retrieval. Endocrinology 153, 4766-4774 (2012).

8. Miller, A. H. Inflammation versus glucocorticoids as purveyors of pathology during stress: have we reached the tipping point? Biol. Psychiatry 64, 263-265 (2008).

9. Miller, G. E. et al. Low early-life social class leaves a biological residue manifested by decreased glucocorticoid and increased proinflammatory signaling. Proc. Natl Acad. Sci. USA 106, 14716-14721 (2009).

10. Cole, S. W. Elevating the perspective on human stress genomics. Psychoneuroendocrinology 35, 955-962 (2010). 